\title{
CONTROLLED CVD SYNTHESIS OF SINGLE-WALLED CARBON NANOTUBES FROM ALCOHOL
}

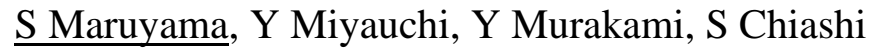 \\ Department of Mechanical Engineering, The University of Tokyo, \\ 7-3-1 Hongo, Bunkyo-ku, Tokyo 113-8656, Japan
}

By using alcohol as carbon source, high-purity single-walled carbon nanotubes (SWNTs) can be generated at relatively low CVD temperatures from metal catalysts supported with zeolite [1]. Based on these findings, we have proposed alcohol catalytic CVD (ACCVD) technique. In addition to the conventional metal particles supported on zeolite, we have developed a simple dip-coat method to directly disperse nano-particles on flat substrates such as quartz and silicon [2]. As shown in Fig. 1, the vertically aligned SWNTs film with about 10 micron meters is grown on quartz substrates by employing the most efficient activation of catalytic metals [3,4]. In addition to molecular dynamics simulations [5] as shown in Fig. 2, various experimental techniques have been employed to understand the growth mechanism of SWNTs: direct TEM observation of catalysts particles [6]; in-situ Raman and AFM measurements during CVD [7]; in-situ monitoring of laser absorption [4]; combinatory spattering method to prepare catalysts [8].

On the other hand, the chirality distribution of SWNTs measured by the near infrared fluorescence spectroscopy is quite unique for low-temperature CVD condition [9] as shown in Fig. 3. The near armchair nanotubes are predominantly generated probably because of the stability of nanotube cap structure. The infrared fluorescence is strong enough to be observed from individual nanotubes on a silicon substrate [10].

The anisotropic optical properties of SWNTs can be intensively studied with the vertically aligned SWNTs or aligned individual SWNTs with gelatine matrix. Polarized resonant Raman [11] and polarized optical absorption [12] studies of vertically aligned SWNTs clearly shows the anisotropic optical properties of nanotubes. Applications of SWNTs in optical devices such as polarizer and saturable absorbers for modelocked fiber lasers [13] are expected.

\section{References}

[1] S. Maruyama et al., Chem. Phys. Lett. 360 (2002) 229.

[2] Y. Murakami et al., Chem. Phys. Lett. 377 (2003) 49.

[3] Y. Murakami et al., Chem. Phys. Lett. 385 (2004) 298.

[4] S. Maruyama et al., Chem. Phys. Lett. 403 (2005) 320.

[5] Y. Shibuta et al., Chem. Phys. Lett. 382 (2003) 381.

[6] M. Hu et al., J. Catal. 225 (2004) 230.

[7] S. Chiashi et al., Chem. Phys. Lett. 386 (2004) 89.

[8] S. Noda et al., Appl. Phys. Lett. 86 (2005)173106.

[9] Y. Miyauchi et al., Chem. Phys. Lett. 387 (2004) 198.

[10] K. Matsuda et al., Appl. Phys. Lett. 86 (2005) 123116.

[11] Y. Murakami et al., Phys. Rev. B 71 (2005) 085403.

[12] Y. Murakami et al., Phys. Rev. Lett. 94 (2005) 087402.

[13] S. Yamashita et al., Opt. Lett. 29 (2004)1581.

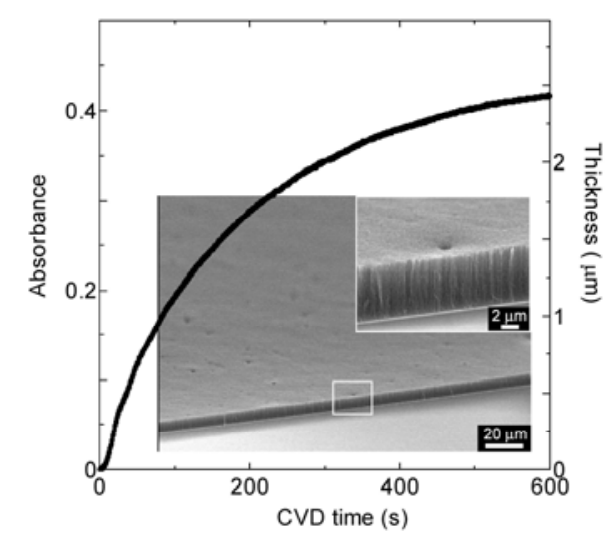

Fig. 1 Growth process of vertically aligned SWNTs

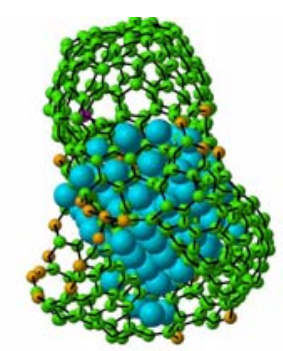

Fig. 2 Molecular dynamics simulation of nucleation of SWNTs

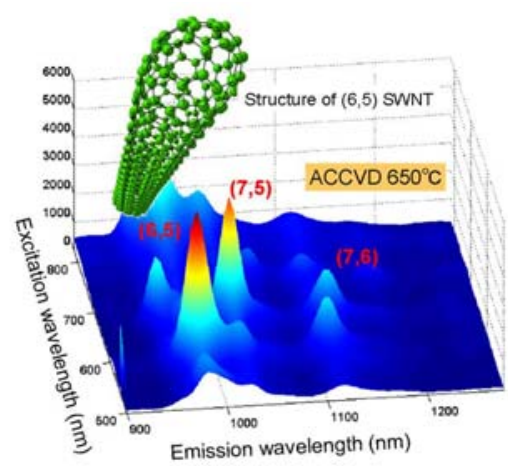

Fig. 3 Near infrared fluorescence of SWNTs and structure of $(6,5)$ nanotube 